\title{
Correlative In-situ Gas and Heating TEM: Integrating Calorimetry and Mass Spectroscopy
}

\author{
Dan Zhou, Ronald G. Spruit, Merijn Pen, Hugo Perez Garza and Qiang Xu \\ DENSsolutions B.V., Delft, Zuid-Holland, Netherlands
}

\section{Introduction}

The profound information provided by TEM, such as the particle size, shape, composition, crystallography, atomic and electronic structure, is fundamental in understanding the structure-property relationship and critical in controlling the atomic-scale synthesis of novel catalysts with improved efficiency. The historical trend of pursuing higher and higher spatial and energy resolution required better and better top-down vacuum conditions inside a TEM ${ }^{1}$. However, this high vacuum contradicts with the desire to study materials in their working conditions, such as gas environment or in liquids ${ }^{2}$. Meanwhile, the correlation during materials' working process is missing. Temporally and spatially resolved in-situ and operando TEM is the only technique can bridge these contradictory but all-important needs and provides insights to the structure-property dynamic relationships at atomic scale ${ }^{3,4}$.

As shown in Figure 1(a), nano-reactors based on microelectromechanical systems (MEMS) ${ }^{5}$ make it possible to increase the pressure around the specimen to more than 1 atmospheric pressure and heat the specimen up to $1000{ }^{0} \mathrm{C}$ while maintaining the high vacuum in the other parts of TEM, i.e. the ex-situ TEM capabilities are conserved. The integration of these nano-reactors with a swift and continuous composition variable gas supply system (GSS) and residual gas analysis (RGA) system, transforms the TEM to a micro-sized chemical plant with on-site structure and chemistry analysis.

In this work, we will present the correlative investigations of catalyst nanoparticles enabled by such insitu gas and heating TEM system, integrating calorimetry and mass spectroscopy, with a case study of the Pd catalyzing methane oxidation processes.

\section{Material and Methods}

DENSsolutions' Climate G+ system, including 3 feeds GSS, TEM sample holder, gas and heating nanoreactor and Impulse software, DENSsolutions gas analyzer, and a FEI Titan (TEM) operated at $300 \mathrm{kV}$ were used in this work. Commercial Pd nanoparticles with an average size of $20 \mathrm{~nm}$ were transferred to the Climate nano-reactor by drop-casting.

\section{Results and Discussions}

Figure 1(b) shows a typical high-angle annular dark-field (HAADF) scanning transmission electron microscopy (STEM) image from a time-series HAADF STEM image stack acquired with oxygen concentration change. Due to the format limitation, the video will be shown on site. The time-series movie started with oxygen to methane 1:1 and decreased as time went by and shows that the nanoparticles movement (dancing) slowed down and finally reached static status. More TEM data, like atomicresolution STEM images, EELS and EDS gives further in-depth structure information. 
Figure 2(a-c) shows the real time changes of catalytic activity as a function of temperature with set oxygen to methane ratio and nano-reactor pressure. Activation of the catalyst was found to be around $420{ }^{0} \mathrm{C}$, as highlighted by the green dashed lines showing the significant increasing production of $\mathrm{CO}_{2}$. And the oscillating behavior of the catalyst was observed to start from a temperature about $700{ }^{0} \mathrm{C}$. Note that the gas species measured by RGA include reaction precursor $\mathrm{CH}_{4}$ and $\mathrm{O}_{2}$, reaction products $\mathrm{CO}, \mathrm{CO}_{2}$ and $\mathrm{H}_{2} \mathrm{O}$, and inert gas $\mathrm{Ar}$ ( $\mathrm{Ar}$ is used to dilute the explosive gas exhausted to the environment for safety considerations). For simplicity, only $\mathrm{CO}_{2}$ was presented here. Enabled by the 4-point-probe microheater design $^{6}$, the heating power consumed is correlate with temperature, gas species and the amount of heat absorbed or dissipated by the specimen during endo- or exothermic reactions, as shown in figure 2(c). The endo- or exothermic reactions' contributions are manifested by the heating power oscillations at constant temperature of $800{ }^{0} \mathrm{C}$. The oscillating catalytic behavior has also been studied as a function of composition, pressure and so on. Further interpretation and more details will be presented.

Through this presentation, we would like to demonstrate the inspiring research possibilities enabled by in situ TEM and introduce the workflow of in situ gas and heating TEM.
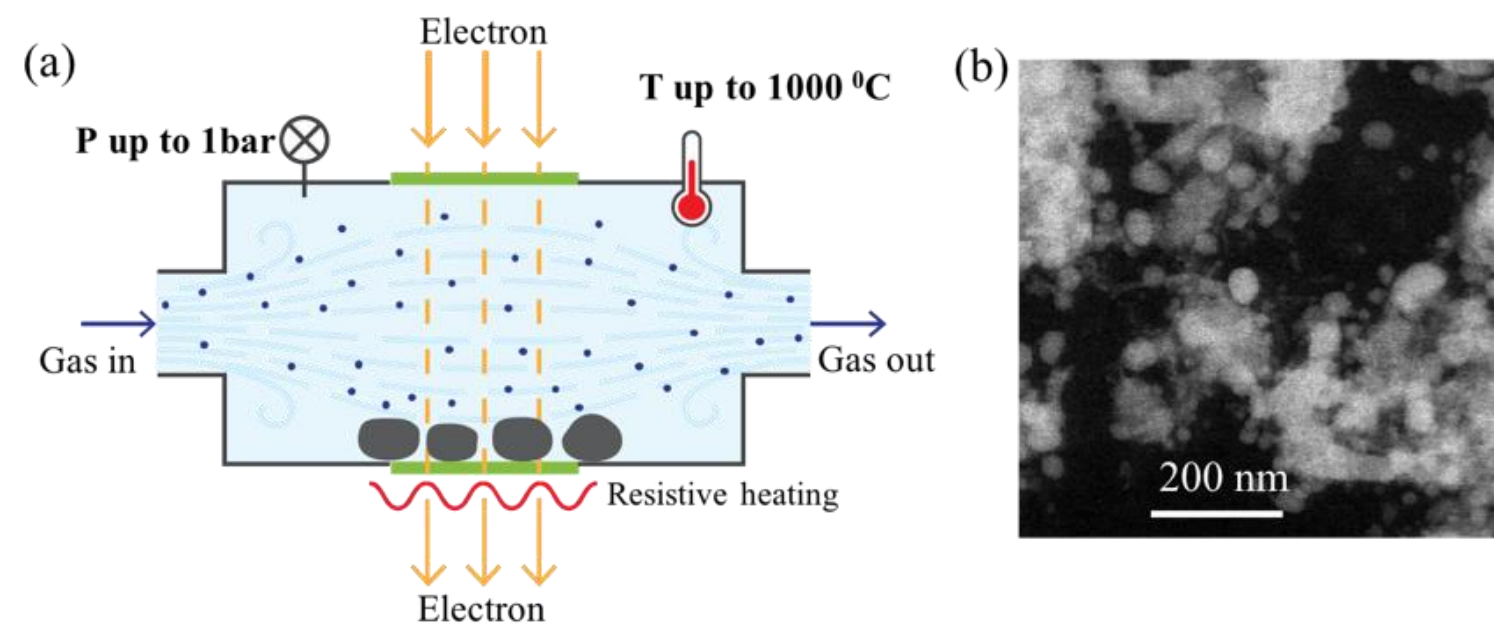

Figure 1. (a) Schematic view of the cross section of a Climate nano-reactor and its functionalities. (b) An HAADF image of Pd nanoparticles from the time-series image stack showing the dynamic movement of these nanoparticles in methane oxidation process. 


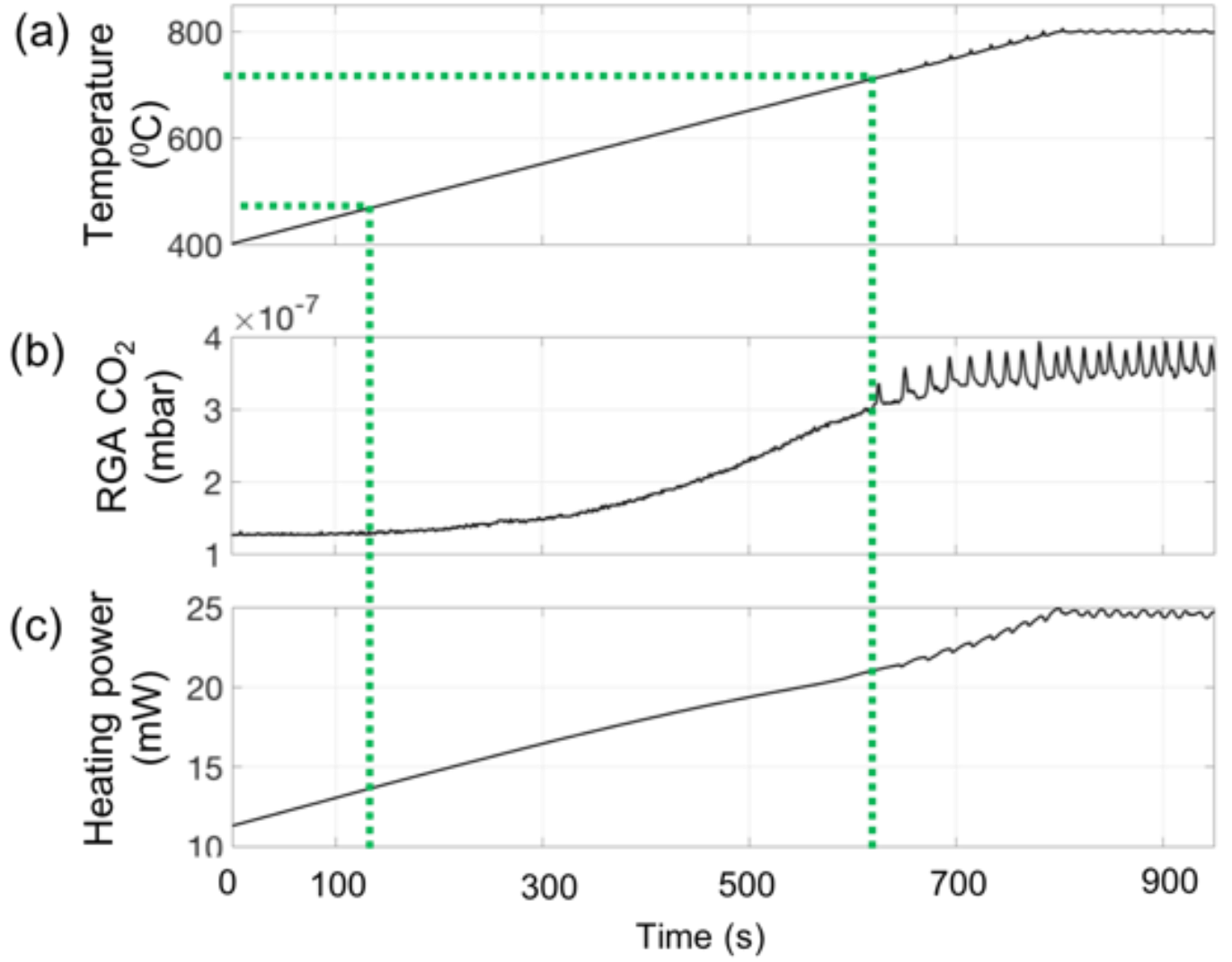

Figure 2. (a-c) Catalytic behavior as a function of temperature. (a) continuous change of temperature from 400 to $800{ }^{0} \mathrm{C}$; (b) The RGA detected reaction product $\mathrm{CO}_{2}$ increases obviously from around $420{ }^{0} \mathrm{C}$ and oscillates from about $700{ }^{0} \mathrm{C}$; (c) The heating power increases with temperature and oscillates with temperature oscillation to maintain required temperature.

\section{References}

1. Brydson, R., Aberration-Corrected Analytical Transmission Electron Microscopy, Wiley, 2011.

2. Hansen, T.W., Wagner, J.B., Controlled Atmosphere Transmission Electron Microscopy: Principles and Practice, Springer, 2016.

3. Plodinec, M.al. Insights into Chemical Dynamics and Their Impact on the Reactivity of Pt Nanoparticles during CO oxidation by Operando TEM, ACS Catalysis 2020,10, 3183-3193.

4. Vendelbo, S.B.al. Visualization of oscillatory behavior of Pt nanoparticles catalyzing CO Oxidation, Nature Materials 2014, 13(9), 884-890.

5. Perez-Garza, H., Morsink, D., Xu, J. Sholkina, M., Pivak, Y., Pen, M., van Weperen, S., and Xu, Q. IEEE-NEMS (2016).

6. https://denssolutions.com/products/climate/ 